\title{
Implementing a Nursing Systems Framework in a developing
}

\section{country}

Ann Marie CANNABY, Professor of Nursing, Birmingham City University, Birmingham, UK

Ioannis GKANTARAS, Independent Consultant, Athens, Greece

Aimee FINN, Independent Consultant, Dublin, Ireland

Brent FOREMAN, Assistant Executive Director, Hamad Medical Corporation, Doha,

Qatar

Gina BUTLER, Assistant Executive Director, Hamad Medical Corporation, Doha, Qatar

Annie TOPPING, Assistant Executive Director, Hamad Medical Corporation, Doha, Qatar and the University of Calgary, Qatar

Richard GRAY, Professor of Clinical Nursing, La Trobe University, Melbourne, Australia

Corresponding author

\section{Richard Gray}

Professor of Clinical Nursing

La Trobe University

Melbourne, Australia

Email: r.gray@latrobe.edu.au 


\section{Implementing a Nursing Systems Framework}

\section{Additional Information}

\section{Funding}

The funding for this research was provided by the Qatar Academic Health System, Health Services and Population Health Research Program. The aim of this program is to stimulate independent and high quality research in areas that affect the health of the population of Qatar.

\section{Resources Accessibility}

Access to the study protocol, the original data, and the programming code implemented to extract and integrate the data or the methodology that was followed to produce the final research dataset is available by request.

\section{Author statement}

AMC, IG and RG conceived the study. AMC, AF, BF, AT GB and RG developed and supported the implementation of the NSF. IG extracted and analysed the data. IG, RG and AMC wrote the first draft of the manuscript. All authors contributed to revising the paper and approved the final version.

\section{Conflicts of interest}

AC, BF, AT, GF, and RG were involved in the development of the Nursing Systems Framework that was evaluated in this study.

Word count: 3,985 